\title{
Modeling of Broadband Spectra and Radial Profiles of Emission of Pulsar Wind Nebulae
}

\author{
Wataru Ishizaki* \\ Yukawa Institute for Theoretical Physics \\ E-mail: wataru.ishizakieyukawa.kyoto-u.ac.ip
}

\section{Katsuaki Asano}

Institute for Cosmin-Ray Research

E-mail: asanokeicrr.u-tokyo.ac.jp

\section{Kyohei Kawaguchi}

Institute for Cosmin-Ray Research

E-mail: kkawadicrr.u-tokyo.ac.jp

\begin{abstract}
X-ray emission of a pulsar wind nebula (PWN) is a group of particles with the highest energy among the non-thermal electron-positrons present in the nebula. The standard emission model of PWNe predicts that such high energy particles lose their energy immediately by radiative cooling and that the emission region of $\mathrm{X}$-rays is smaller than those of lower frequencies. However, as $3 \mathrm{C}$ 58 and G21.5-0.9, some PWNe have been discovered in which the emission region of X-rays and radio extend to the almost same extent, and it has been pointed out that the conventional model has room for reconsideration. We investigate the validity of the model by simultaneously calculating the emission spectrum integrating over the entire nebula and the radial profile of the X-ray surface brightness. Our detailed analysis reveals that the conventional model cannot explain observational facts of 3C 58 and G21.5-0.9. Furthermore, in order to improve the model, we construct a model of PWNe in consideration of spatial diffusion due to a disturbed magnetic field. We show that entire spectra and X-ray surface brightness profiles are reproduced simultaneously by our new model. The model implies the existence of a large gamma-ray halo which consists of particles diffusely escaped out of the nebula. The halo could spread to a degree that it can be resolved spatially by the Cherenkov Telescope Array.
\end{abstract}

36th International Cosmic Ray Conference -ICRC2019-

July 24th - August 1st, 2019

Madison, WI, U.S.A.

${ }^{*}$ Speaker. 


\section{Introduction}

A rotation-driven pulsar emits its rotational energy as a relativistic plasma flow, which is called a pulsar wind. It interacts with a supernova remnant or interstellar matter around the pulsar, and forms a bubble-like structure accompanying a strong shock, which is called the pulsar wind nebula (PWN). Electrons and positrons injected to the nebula are accelerated with the shock. They emit broadband emission from radio to gamma-ray via the synchrotron radiation and inverse Compton scattering (ICS). In particular, X-ray photons are emitted by synchrotron radiation from the most energetic particles present in the nebula. Thus, the spatial distribution of X-ray emission of the PWN contains information on the propagation processes of the most energetic particles in the nebula.

A standard model of PWNe was established by Kennel and Coroniti (hereafter KC84) [ [1, [2]. They modeled the dynamical structure of the nebula with a one-dimensional steady-state magnetohydrodynamic model [四], and calculated synchrotron emission consistently on that [2]. Their model (hereafter KC model) was applied to the Crab nebula and explained the following three observations: the fact that the pulsar wind, which has flowed in at the speed of light on the termination shock, is decelerating to $O(1000) \mathrm{km} \mathrm{s}^{-1}$ near the edge of the nebula, the emission spectrum integrated over the entire nebula, and, the tendency that the extent of the emission region becomes smaller as the higher frequency band. Their model was extended by Atoyan and Aharonian [B] as a model considering the inverse Compton scattering and established itself as a standard model that well reproduces the observed features of the Crab nebula.

Thanks to the detail observation of many of pulsar wind nebulae by Chandra Observatory, it has been found that there is room for reconsideration in KC model. In fact, for 3C 58 [䧃] and G21.5-0.9 [5], it was discovered that the extent of the X-ray emission is comparable to the radio one, although the age of each PWNe is about the same as the Crab nebula. In contrast, in the Crab Nebula, the diameter of the radiation area of X-rays of several $\mathrm{keV}$ is about half smaller than that of lower frequency bands such as optical and radio bands. This led to the recognition that PWNe model needs to be improved. For example, some authors [G, $\square]$ constructed a model that can explain the spread of the extent of X-ray nebula by incorporating the diffusion process that has not been considered in the $\mathrm{KC}$ model into the model. However, there are no studies that clarified whether this X-ray spread problem is an observation fact that can not be really explained within the scope of the $\mathrm{KC}$ model. In order to clarify the problems of the $\mathrm{KC}$ model, we examine both the emission spectrum integrated over the entire nebula and the surface brightness profile of X-rays simultaneously [ [8]. Furthermore, based on the results, we construct a model with the diffusion process, and the observed entire spectra and surface brightness profiles are simultaneously reproduced for G21.5-0.9 and 3C58 [9]. These are based on the results reported as Ishizaki et al. (2017) [8] and Ishizaki et al. (2018) [0].

\section{Method}

Our methodology is mainly established by Ishizaki et al.(2017,2018) [ [8], Q]. We assume that the PWN is a steady and spherical bubble with a radius $r_{\mathrm{N}}$. The pulsar wind is emitted by the central pulsar and forms a strong termination shock at a radius $r_{\mathrm{s}}$. Assuming that the pre-shock wind is 
cold and the magnetic field is purely toroidal, we are able to characterize the wind physical property by three quantities: the comoving number density $n$, bulk Lorentz factor $\gamma$, and magnetic field in the lab frame $B$ at the shock front. The pulsar spin-down luminosity $L_{\text {sd }}$ is almost all converted to pulsar wind, namely

$$
L_{\mathrm{sd}}=4 \pi r_{\mathrm{s}}^{2} n_{\mathrm{u}} u_{\mathrm{u}} \gamma_{\mathrm{u}} m_{\mathrm{e}} c^{3}(1+\sigma)
$$

where $u=\gamma \beta$ is a radial four velocity, subscript $u$ (upstream) denotes the value of quantities just before shock, and $\sigma$ is a magnetization parameter, which is written as

$$
\sigma \equiv \frac{B_{\mathrm{u}}^{2} / 4 \pi}{n_{\mathrm{u}} u_{\mathrm{u}} \gamma_{\mathrm{u}} m_{\mathrm{e}} c^{2}} .
$$

Our calculation method mainly consists of the following steps:

1. Obtaining the hydrodynamic structure of the nebula by solving the spherically symmetric steady MHD equations.

2. As a boundary condition at $r_{\mathrm{s}}$, assuming that the non-thermal energy distribution of electrons and positrons. Calculating the energy spectrum of particles at each radius with this boundary condition.

3. Calculating the emissivity at each radius from the energy spectrum, and calculate the observable quantities (i.e. entire spectrum and surface brightness) with consideration about the geometry.

Hereafter, although we introduce two models, the $\mathrm{KC}$ model and our new model taking into account the diffusion process (hereafter DF model), the calculation procedure is basically the same. Note that, with regard to DF model, in order to satisfy the consistency between the fluid structure and the distribution function of non-thermal particles, we formulated the effect of the back-reaction of the diffusion process to the fluid motion. However, in the scope of the results presented in this paper, the effect of the back-reaction is almost negligible, so we omit a description of that formulation (see Ishizaki et al.(2018)[Q] for details).

For the fluid structure, the almost same calculation as the $\mathrm{KC}$ model [W] is mainly performed. Assuming that the flow direction is purely radial, that the magnetic field is perpendicular to the flow direction, and the flow is adiabatic, we solve the spherically symmetric steady MHD equation. The boundary condition adopts the Rankine-Hugoniot condition at $r_{\mathrm{s}}$. According to the KC84, the flow velocity just downstream of the termination shock can be written as,

$$
u_{\mathrm{d}}^{2}=\frac{8 \sigma^{2}+10 \sigma+1+\sqrt{64 \sigma^{2}(\sigma+1)^{2}+20 \sigma(\sigma+1)+1}}{16(\sigma+1)} .
$$

The magnetic field at upstream (subscript $u$ ) and downstream (subscript $d$ ) is written as,

$$
\begin{gathered}
B_{\mathrm{u}}=\left[\frac{L_{\mathrm{sd}}}{c r_{\mathrm{s}}^{2}} \frac{\sigma}{1+\sigma}\right]^{1 / 2}, \\
B_{\mathrm{d}}=B_{\mathrm{u}} \frac{\gamma_{\mathrm{d}}}{u_{\mathrm{d}}}
\end{gathered}
$$


After some algebra, the following equation for the downstream flow profile can be obtained from MHD equations (see KC84 for details),

$$
\sqrt{1+u^{2}(r)}\left(\delta+\frac{\left(u_{\mathrm{d}}^{2} / \sigma\right)-\frac{1}{2}}{u_{\mathrm{d}}^{2}+\frac{1}{4}}\left(\frac{u(r) r^{2}}{u_{\mathrm{d}} r_{\mathrm{s}}^{2}}\right)^{-\frac{1}{3}}+\frac{u_{\mathrm{d}}}{u(r)}\right)=\gamma_{\mathrm{d}}\left(\delta+\frac{\left(u_{\mathrm{d}}^{2} / \sigma\right)-\frac{1}{2}}{u_{\mathrm{d}}^{2}+\frac{1}{4}}+1\right),
$$

where $\gamma=\sqrt{1+u^{2}}$ is a bulk Lorentz factor, and $\delta=u_{\mathrm{d}} /\left(\sigma u_{\mathrm{u}}\right)$ is a constant which is much smaller than unity in the standard parameter range. We solve equation (2.6) numerically. Using the obtained velocity field $u(r)$, the spatial structure of the magnetic field can be calculated as follows from the conservation law of the magnetic flux,

$$
B(r)=B_{\mathrm{d}} \frac{\gamma(r)}{\gamma_{\mathrm{d}}} \frac{u_{\mathrm{d}} r_{\mathrm{s}}}{u(r) r} .
$$

Next, the equations describing the energy spectrum $n(r, E)$ of the particles are solved along the flow. The transport equation of relativistic pairs is given by

$$
\frac{1}{r^{2}} \frac{\partial}{\partial r}\left[r^{2}\left(c u(r) n(r, E)-\kappa \frac{\partial n(r, E)}{\partial r}\right)\right]-\frac{\partial}{\partial E}[Q(r, E) n(r, E)]=0,
$$

where $\kappa$ is a diffusion coefficient, $Q$ is energy loss rate per particle, namely

$$
Q=Q_{\mathrm{rad}}+\frac{E}{3} \frac{c}{r^{2}} \frac{\partial}{\partial r}\left[r^{2} u(r)\right]
$$

first term $Q_{\text {rad }}$ represents the energy loss via synchrotron radiation and ICS, and second term represents the energy loss via adiabatic cooling. For the case of KC model and DF model, we adopt $\kappa=0$ and $\kappa \propto E^{1 / 3}$ respectively. At the termination shock $r=r_{\mathrm{s}}, n\left(E, r_{\mathrm{s}}\right)$ is assumed to have following broken power-law shape:

$$
n\left(E, r_{\mathrm{s}}\right)=\left\{\begin{array}{ll}
\frac{n_{0}}{E_{\mathrm{b}}}\left(\frac{E}{E_{\mathrm{b}}}\right)^{-p_{1}} & \left(E_{\min }<E<E_{\mathrm{b}}\right) \\
\frac{n_{0}}{E_{\mathrm{b}}}\left(\frac{E}{E_{\mathrm{b}}}\right)^{-p_{2}} & \left(E_{\mathrm{b}}<E<E_{\max }\right)
\end{array},\right.
$$

where $n_{0}$ is a normalization factor which determined by consistency with fluid quantities, $E_{\min }$ is the minimum energy of particles, $E_{\mathrm{b}}$ is a energy which corresponds to the spectral break introduced phenomenologicaly, $E_{\max }$ is a maximum energy of particles, and two power law indices $p_{1}$ and $p_{2}$ for low and high energy particles. Since the minimum energy $E_{\min }$ hardly affect the resultant observable (i.e. entire spectrum), it is difficult to constrain this value from observation, so we fix the value of the minimum energy $E_{\min }=10 m c^{2}$. The maximum energy $E_{\max }$ of the particle is determined by the Hillas condition at termination shock, namely

$$
E_{\max }=e B_{\mathrm{u}} r_{\mathrm{s}}=\sqrt{\frac{e^{2}}{c} \frac{L_{\mathrm{sd}} \sigma}{1+\sigma}} .
$$

Power-law indices $p_{1}$ and $p_{2}$ are basically free parameters, but $p_{1}$ is well obtained by radio observation. We calculated the energy spectrum of the particle at each radius by solving equation (2.8) numerically with equation ([2.10) as the boundary condition. 
Table 1: Parameters in our calculations.

\begin{tabular}{|c|c|c|c|c|c|}
\hline \multirow[b]{2}{*}{ Given Parameters } & \multirow[b]{2}{*}{ Symbol } & \multicolumn{2}{|c|}{$3 \mathrm{C} 58$} & \multicolumn{2}{|c|}{ G21.5-0.9 } \\
\hline & & $\mathrm{KC}$ & DF & $\mathrm{KC}$ & DF \\
\hline Spin-down Luminosity (erg s ${ }^{-1}$ ) & $L_{\mathrm{sd}}$ & \multicolumn{2}{|c|}{$3.0 \times 10^{37}$} & \multicolumn{2}{|c|}{$3.5 \times 10^{37}$} \\
\hline Distance $(\mathrm{kpc})$ & $D$ & \multicolumn{2}{|c|}{$2.0^{\mathrm{a}}$} & \multicolumn{2}{|c|}{$4.8^{\mathrm{b}}$} \\
\hline Radius of the nebula (pc) & $r_{\mathrm{N}}$ & \multicolumn{2}{|c|}{2.0} & \multicolumn{2}{|c|}{0.9} \\
\hline \multicolumn{6}{|l|}{ Fitting Parameters } \\
\hline Break Energy (eV) & $E_{\mathrm{b}}$ & $4.1 \times 10^{10}$ & $3.0 \times 10^{10}$ & $2.6 \times 10^{10}$ & $6.0 \times 10^{10}$ \\
\hline Low-energy power-law index & $p_{1}$ & 1.26 & 1.08 & 1.1 & 1.2 \\
\hline High-energy power-law index & $p_{2}$ & 3.0 & 2.9 & 2.3 & 2.5 \\
\hline Radius of the termination shock (pc) & $r_{\mathrm{s}}$ & 0.13 & 0.14 & 0.05 & 0.05 \\
\hline Magnetization parameter & $\sigma$ & $1.0 \times 10^{-4}$ & $2.0 \times 10^{-4}$ & $2.0 \times 10^{-4}$ & $6.0 \times 10^{-4}$ \\
\hline Diffusion coefficient at $E_{\mathrm{b}}\left(\mathrm{cm}^{2} \mathrm{~s}^{-1}\right)$ & $\kappa_{0}$ & - & $1.0 \times 10^{26}$ & - & $1.0 \times 10^{26}$ \\
\hline \multicolumn{6}{|l|}{ Obtained Parameters } \\
\hline Initial bulk Lorentz factor & $\gamma_{u}$ & $7.3 \times 10^{3}$ & $2.4 \times 10^{4}$ & $2.1 \times 10^{4}$ & $1.9 \times 10^{4}$ \\
\hline Pre-shock magnetic field $(\mu \mathrm{G})$ & $B_{\mathrm{u}}$ & 0.79 & 1.0 & 3.1 & 5.4 \\
\hline Maximum energy $(\mathrm{eV})$ & $E_{\max }$ & $9.5 \times 10^{13}$ & $1.3 \times 10^{14}$ & $1.4 \times 10^{14}$ & $2.5 \times 10^{14}$ \\
\hline Average magnetic field $(\mu \mathrm{G})$ & $B_{\text {ave }}$ & 31 & 34 & 120 & 133 \\
\hline
\end{tabular}

a [ए]]; ${ }^{\mathrm{b}}$ [ए2].

Finally, we calculate the emission via synchrotron radiation and ICS from non-thermal particles. The spectrum of synchrotron radiation can be calculated by using the value of the magnetic field at each point calculated by equation (2.7). In order to calculate the spectrum of ICS, the result of GALPROP v54.1 [प0, [1] was adopted as the spectrum of the seed photon. The emissivity $j_{v}$ at each radius can be obtained by using the solution of equation (2.8) at each radius. The entire spectrum is obtained by integrating $j_{v}$ over the entire nebula, namely

$$
F_{v}=\frac{1}{D^{2}} \int_{r_{\mathrm{s}}}^{r_{\mathrm{N}}} j_{v}(r) r^{2} d r
$$

where $D$ is a distance to the nebula. The surface brightness can be obtained by integrating $j_{v}$ along the line of sight, namely

$$
B_{v}(s)=2 \int_{\max \left(r_{s}, s\right)}^{r_{\mathrm{N}}} \frac{j_{v}(r) r d r}{\sqrt{r^{2}-s^{2}}},
$$

where $s$ is the distance perpendicular to the line of sight from the central pulsar.

\section{Results}

We adopted 3C 58 and G21.5-0.9 for model verification. See Ishizaki et al. (2017) [8] for details of these objects. In these objects, it has been reported that the extent of the X-ray emission region is comparable to that of radio.

The results of applying our model are summarized in Table $\mathrm{W}$. In the case of $\mathrm{KC}$ model, the results of searching for parameters that can reproduce the entire spectrum of nebula are shown. In the DF model, we choose to explain the spectrum as well, and search for parameters that make the 

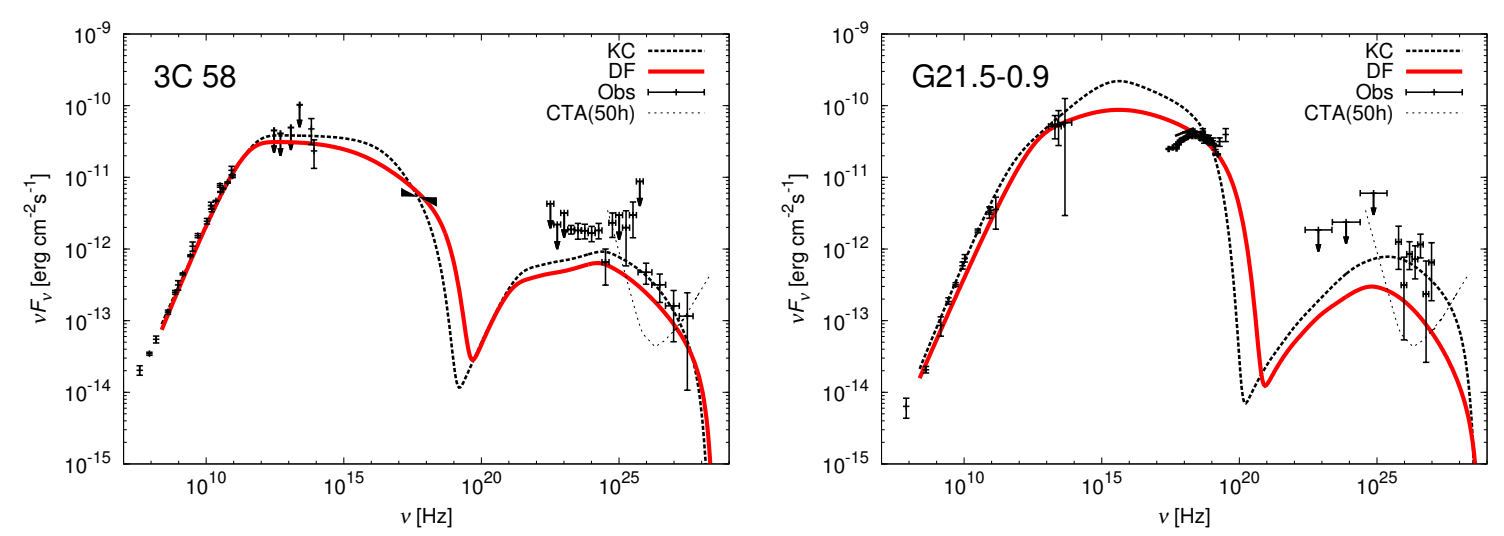

Figure 1: Entire photon spectra for 3C 58 (left panel) and G21.5-0.9 (right panel). The red line represents the DF model, and the black line represents the KC model. See Ishizaki et al.(2018) [G] for details on data points.
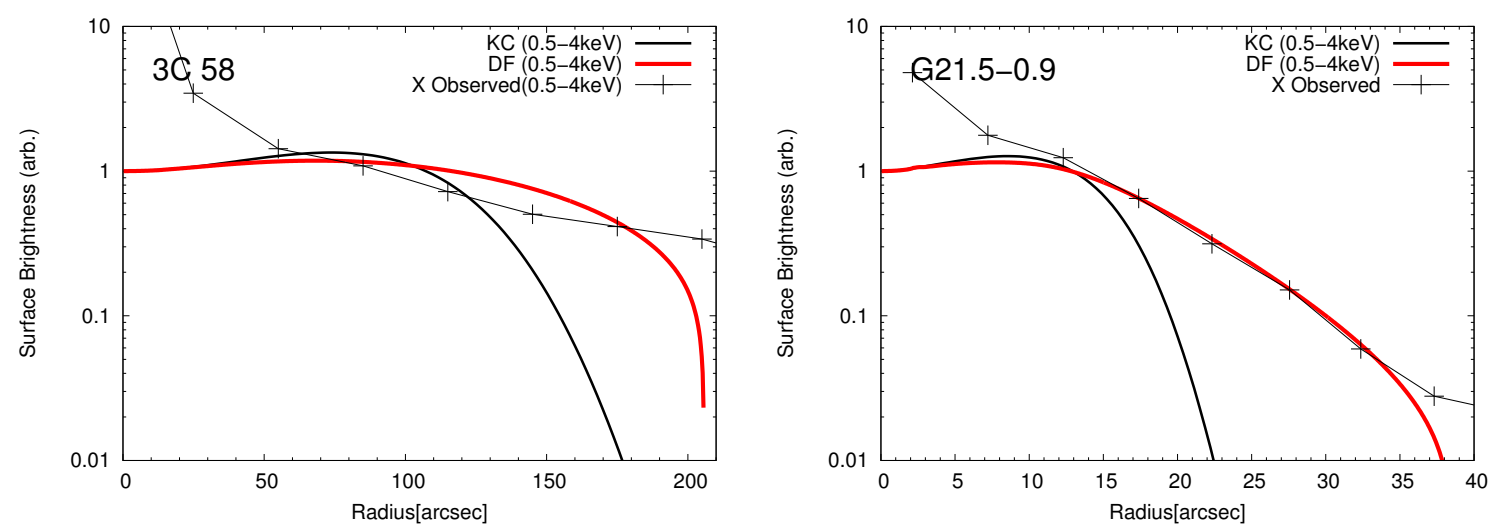

Figure 2: Radial profiles of the surface brightness of X-rays. The red lines represent the DF model, and the thin black solid line represent the X-ray profile of the KC model. All curves are normalized as unity at the center. In both plots, the right end of the $\mathrm{X}$-axis corresponds to the edge of the nebula. The data points are taken from [四] (X) for 3C 58, and [G] (X) for G21.5-0.9.

X-ray spectrum harder. As shown in Figure $\mathbb{W}$, both models are able to explain the entire spectra well. Figure $\square$ shows surface brightness profiles of $\mathrm{X}$-ray calculated under parameters that can explain the entire spectra. For both objects, the calculated curves of the KC model show that the extent of X-ray is smaller that the edge of the nebula (i.e. the extent of the radio nebula). On the other hand, in the DF model, the emission region of X-rays is sufficiently extended to the edge of the nebula for both objects. Figure 3 shows the radial dependence of the X-ray spectral index. In $\mathrm{KC}$ model, sudden softening is observed at the same radius as the X-ray surface brightness decreases. Especially in the case of G21.5-0.9, this behavior clearly contradicts the observational fact. On the other hand, in the DF model, such problematic softening has been eliminated. 

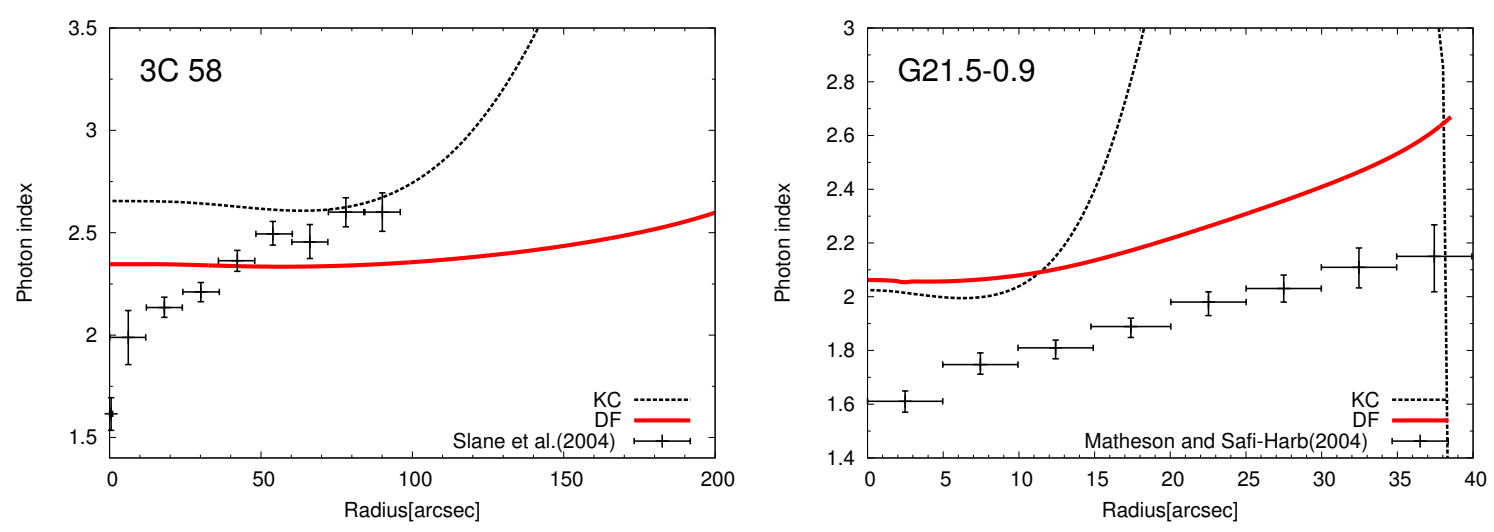

Figure 3: Radial profiles of the photon indices in $0.5-10.0 \mathrm{keV}$ range in the DF model (red), and the KC model (black). The data points are taken from [四] and [G] for 3C 58 and G21.5-0.9, respectively.

\section{Discussion and Summary}

Ishizaki et al. (2017) [[] showed that there is only one parameter set that can explain the entire spectrum by examining the parameter dependence of the $\mathrm{KC}$ model in detail. Furthermore, it is also shown that taking parameters that can reproduce surface brightness can not explain lowerfrequency emission than optical-band of the entire spectrum. This indicates that in KC model, it is not possible to make both the entire spectrum and the surface brightness compatible. On the other hand, in the DF model, both the entire spectrum and the X-ray surface brightness are simultaneously reproduced. However, some issues still remain: The radial dependence of the X-ray spectral index is qualitatively consistent with the observation but is not reproducible quantitatively. Furthermore, as shown in Figure $\mathbb{U}$, the $\mathrm{TeV} \gamma$-ray flux of the entire spectrum is about 2 times smaller than the observation. The latter can be explained by considering ICS from the particles escaped from the nebula diffusively. Although rough estimation, assuming that the obtained diffusion coefficient is the same inside and outside the nebula, the extent of $\mathrm{TeV} \gamma$-ray is about twice the extent of radio nebula. It can be estimated that the diameter of TeV nebula of 3C 58 is 800 " and that of the G21.50.9 is about 160 ". These may be spatially resolved by current imaging atmospheric Cherenkov telescopes or Cherenkov Telescope Array. In the near future, very high-energy $\gamma$-ray observations with high spatial resolution will allow us to quantitatively discuss the escape of particles from PWNe.

\section{References}

[1] Kennel, C. F. and F. V. Coroniti, Confinement of the Crab pulsar's wind by its supernova remnant., ApJ, 1984 (283) 694-709.

[2] Kennel, C. F. and F. V. Coroniti, Magnetohydrodynamic model of Crab nebula radiation., ApJ, 1984 (283) 710-730.

[3] Atoyan, A. M. and F. A. Aharonian, On the mechanisms of gamma radiation in the Crab Nebula, MNRAS, 1996 (278) 525-541. 
[4] Slane, P., D. J. Helfand, E. van der Swaluw, and S. S. Murray, New Constraints on the Structure and Evolution of the Pulsar Wind Nebula 3C 58, ApJ, 2004 (616) 403-413.

[5] Matheson, H. and S. Safi-Harb, The plerionic supernova remnant G21.5-0.9: In and out, AdSpR, 2005 (35) 1099-1105.

[6] Tang, X. and R. A. Chevalier, Particle Transport in Young Pulsar Wind Nebulae, ApJ, 2012 (752) 83.

[7] Porth, O., M. J. Vorster, M. Lyutikov, and N. E. Engelbrecht, Diffusion in pulsar wind nebulae: an investigation using magnetohydrodynamic and particle transport models, MNRAS, 2016 (460) 4135-4149.

[8] Ishizaki, W., S. J. Tanaka, K. Asano, and T. Terasawa, Broadband Photon Spectrum and its Radial Profile of Pulsar Wind Nebulae, ApJ, 2017 (838) 142.

[9] Ishizaki, W., K. Asano, and K. Kawaguchi, Outflow and Emission Model of Pulsar Wind Nebulae with the Back Reaction of Particle Diffusion, ApJ, 2018 (867) 141.

[10] Vladimirov, A. E., S. W. Digel, G. Jóhannesson, P. F. Michelson, I. V. Moskalenko, P. L. Nolan, E. Orlando, T. A. Porter, and A. W. Strong, GALPROP WebRun: An internet-based service for calculating galactic cosmic ray propagation and associated photon emissions, CoPhC, 2011 (182) 1156-1161.

[11] Porter, T. A. and A. W. Strong, A new estimate of the Galactic interstellar radiation field between O.1um and 1000um, ICRC, 2005 (4) 77.

[12] Tian, W. W. and D. A. Leahy, The distances of supernova remnants Kes 69 and G21.5-0.9 from HI and ${ }^{13}$ CO spectra, MNRAS, 2008 (391) L54-L58.

[13] Kothes, R., Distance and age of the pulsar wind nebula 3C 58, A\&A, 2013 (560) A18. 